\title{
Outlining the Scaling-Based and Scaling-Free Optimization of Electrocatalysts
}

\author{
Nitish Govindarajan, ${ }^{\dagger}$ Marc T. M. Koper, ${ }^{\ddagger \oplus}$ Evert Jan Meijer, ${ }^{\dagger}{ }^{\circledR}$ and Federico Calle-Vallejo ${ }^{*}, \S^{\circledR}$ \\ ${ }^{\dagger}$ Amsterdam Center for Multiscale Modeling and Van 't Hoff Institute for Molecular Sciences, Science Park 904, 1098 XH, \\ Amsterdam, The Netherlands \\ ${ }^{\ddagger}$ Leiden Institute of Chemistry, Leiden University, P.O. Box 9502, 2300 RA Leiden, The Netherlands \\ ${ }^{\S}$ Departament de Ciència de Materials i Química Fisica, Institut de Química Teòrica i Computacional (IQTCUB), Universitat de \\ Barcelona, Martí i Franqués 1, 08028 Barcelona, Spain
}

\section{Supporting Information}

ABSTRACT: For nearly 15 years, significant efforts have been directed toward the computational design of electrocatalysts for a variety of important reactions. Despite conspicuous discoveries, enhancing electrocatalysts is still a feat rather than a routine task. This could be due to the fact that computational materials design is often guided by heuristic rules. Here we outline a systematic procedure for the optimization of electrocatalysts using two independent parameters: $\delta$, which is restricted by adsorption-energy scaling relations, and $\varepsilon$, which is scaling-free. Taking the prototypical oxygen evolution reaction as a case study, we mathematically show that, contrary to the widespread idea, stabilizing $* \mathrm{OOH}$ with respect to $* \mathrm{OH}$ is not a universal principle to go beyond the top of the activity volcano. Conversely, the $\delta-\varepsilon$ optimization lowers the calculated overpotentials in nearly all analyzed

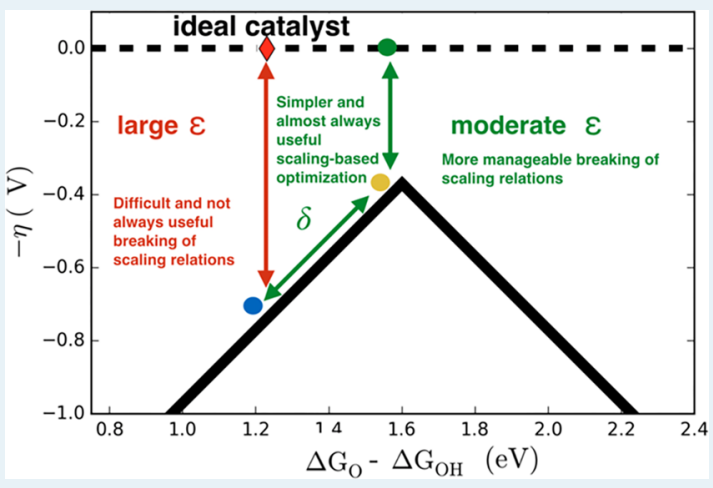
cases, suggesting that "electrocatalytic symmetry" is the only general thermodynamic recipe for optimal electrocatalysis. Using $\delta-\varepsilon$ analyses, screening studies can identify (1) the most promising materials, (2) the problematic reaction intermediates, and (3) the materials' ease of optimization.

KEYWORDS: scaling relations, catalyst optimization, oxygen evolution, electrochemical-step symmetry index, volcano-type activity plot, density functional theory

\section{INTRODUCTION}

Fuel cells and electrolyzers hold promise as the cornerstone of sustainable energy-provision schemes. ${ }^{1}$ However, the global implementation of such schemes might only be triggered by substantial improvements in the activity, selectivity, and stability of the electrocatalysts used in such devices. ${ }^{2}$ Around a decade ago, Nørskov, Rossmeisl and co-workers devised a robust framework based on thermodynamics ${ }^{3}$ and activity descriptors ${ }^{4}$ for the computational assessment of electrocatalytic reactions. Descriptor-based analyses (DBAs) help not only in rationalizing but also in predicting highly active electrocatalytic materials. 5,6

Interestingly, the framework is based on the adsorption energies of surface intermediates and the fact that, in some cases, they exhibit "scaling relations", in particular for similar adsorbates. Scaling relations linearly correlate the adsorption free energies of two species over a set of materials $j: \Delta G_{2}^{j}=A$. $\Delta G_{1}^{j}+B$, where $A$ and $B$ are constants that depend on the adsorbates and/or the geometric configuration of the adsorption sites. $^{7-11}$

If $m$ adsorbates participate in a reaction pathway, the corresponding DBA has $m$ degrees of freedom. However, if $p$ independent scaling relations exist among the $m$ adsorbates, the degrees of freedom are $m-p$ (generally, $m-p \geq 1$ ). Thus, the existence of scaling relations among adsorbates has two farreaching implications for the design of electrocatalysts: (i) DBAs can be greatly simplified, especially for multielectron reactions, owing to the reduction in the degrees of freedom compared with the number of adsorbed species. (ii) The full optimization of electrocatalysts may not be possible because the interdependence of the adsorption energies prevents it. ${ }^{12}$ In brief, (i) and (ii) indicate that scaling relations streamline materials design but also evidence intrinsic limitations in the efficiency of electrocatalysts.

This was first observed in the context of the oxygen evolution reaction (OER, $2 \mathrm{H}_{2} \mathrm{O} \leftrightarrows \mathrm{O}_{2}+4 \mathrm{H}^{+}+4 \mathrm{e}^{-}$) and its reverse reaction, the oxygen reduction reaction $\left(\mathrm{ORR}, \mathrm{O}_{2}+4 \mathrm{H}^{+}+4 \mathrm{e}^{-}\right.$ $\left.\leftrightarrows 2 \mathrm{H}_{2} \mathrm{O}\right){ }^{13}$ These reactions, which are key for the production and oxidation of hydrogen in water electrolyzers and fuel cells, have a standard equilibrium potential of $4.92 \mathrm{eV} / 4 \mathrm{e}^{-}=1.23 \mathrm{~V}$,

Received: February 4, 2019

Revised: $\quad$ March 25, 2019

Published: March 28, 2019 
but they require considerable overpotentials to proceed. For the OER, Rossmeisl et al. proposed the following pathway: ${ }^{14}$

$$
\begin{aligned}
& \mathrm{H}_{2} \mathrm{O}+* \rightarrow * \mathrm{OH}+\mathrm{H}^{+}+\mathrm{e}^{-} \\
& * \mathrm{OH} \rightarrow * \mathrm{O}+\mathrm{H}^{+}+\mathrm{e}^{-} \\
& * \mathrm{O}+\mathrm{H}_{2} \mathrm{O} \rightarrow * \mathrm{OOH}+\mathrm{H}^{+}+\mathrm{e}^{-} \\
& * \mathrm{OOH} \rightarrow *+\mathrm{O}_{2}+\mathrm{H}^{+}+\mathrm{e}^{-}
\end{aligned}
$$

If eqs 2 and 3 are summed up $\left(* \mathrm{OH}+\mathrm{H}_{2} \mathrm{O} \rightarrow * \mathrm{OOH}+2 \mathrm{H}^{+}+\right.$ $\left.2 \mathrm{e}^{-}\right)$, one finds that $* \mathrm{OOH}$ and $* \mathrm{OH}$ are separated by two proton-electron transfers, so that the adsorption energies must differ by $1.23 \mathrm{~V} \times 2 \mathrm{e}^{-}=2.46 \mathrm{eV}$ on ideal catalysts. Instead, the difference is found to be nearly $3.2 \mathrm{eV}$ for a large number of different materials, namely metals, (mixed) oxides, graphitic materials, porphyrins, and so on. ${ }^{15-21}$ The difference between those two values has been repeatedly used to rationalize the inefficiency of electrocatalysts not only for the OER but also for the ORR. It is thus often stated that conventional "oxygen electrodes" possess an "intrinsic" (i.e., material-independent) overpotential due to the unideal energetic separation of $* \mathrm{OH}$ and $* \mathrm{OOH}$, which is as large as $\eta_{\text {intrinsic }}=(3.2-2.46) \mathrm{eV} / 2 \mathrm{e}^{-}=$ $0.37 \mathrm{~V}$ (this corresponds to the top of the volcano in Figure 2). ${ }^{15,18,22-24}$

Ever since this intrinsic, scaling-based overpotential was proposed, extensive computational research has focused on finding electrocatalysts that break or circumvent the scaling relation between $* \mathrm{OH}$ and $* \mathrm{OOH}$. Unfortunately, based on a compendium of literature data, Nørskov and co-workers recently argued that only modest improvements in electrocatalytic activity for the ORR have been made in the past decade, presumably due to the existence of the $\mathrm{OH}-\mathrm{OOH}$ scaling relation. ${ }^{25}$ Judging by recent literature reviews, one could argue that the conclusion for the OER is probably identical. ${ }^{2,26}$ The concept of scaling-based overpotentials has also been extended to other multi- electron reactions like $\mathrm{CO}_{2}$ reduction, where the $\mathrm{CO}-\mathrm{CHO}$ scaling is supposedly responsible for the large overpotentials observed in experiments. ${ }^{27,28}$

While a number of articles focus on breaking the $\mathrm{OH}-\mathrm{OOH}$ scaling relation as a heuristic design principle to optimize OER/ ORR electrocatalysts, ${ }^{18,29-32}$ we note that no study has so far analyzed the precise conditions necessary for such breaking to effectively lower the overpotential. Although such a study is possible using DBAs, there is a methodological void in the literature regarding the optimization of electrocatalysts. In this context, one may ask whether our current difficulties in optimizing electrocatalysts could be (partly) because we rely on rules of thumb instead of having clear mathematical guidelines.

Here we provide material-specific guidelines for the enhancement of electrocatalytic activities based on DBAs. By distinguishing between scaling-based and scaling-free optimization, we show a two-step procedure that provides a quantitative estimation of the possible increase in the activity for a given electrocatalyst. To this end, we introduce the parameters $\delta$ and $\varepsilon$, which indicate the extent of activity optimization with and without the restrictions imposed by scaling relations, respectively.

Although we use the OER catalyzed by a variety of materials (Figure 1) as a case study, we note that the analysis is general and can be applied to any electrocatalytic reaction. Importantly, the
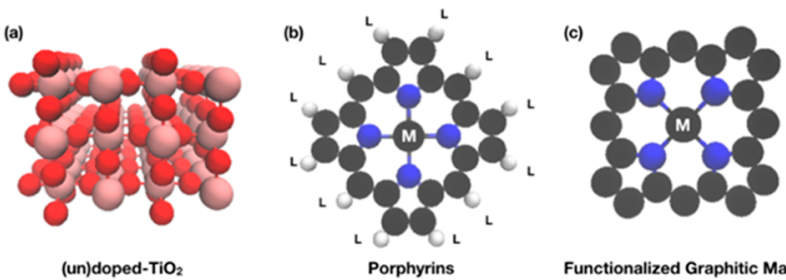

Functionalized Graphitic Materials

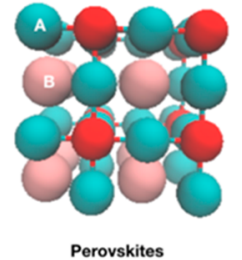

(e)

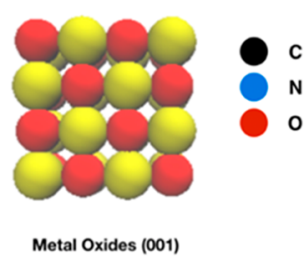

Figure 1. Structures of the catalyst materials used in this study (139 active sites across five classes of materials). (a) 55 doped and undoped $\mathrm{TiO}_{2}$ (110), denoted $\mathrm{d}-\mathrm{TiO}_{2}$. (b) 36 porphyrins, denoted $\mathrm{M}-\mathrm{L}$, where $\mathrm{M}$ is the metal center and $\mathrm{L}$ is the ligand. (c) 13 functionalized graphitic materials (FGMs), denoted M-FGM where $\mathrm{M}$ is the metal center. (d) 24 (100) surfaces of cubic perovskites, denoted $\mathrm{ABO}_{3}$. (e) 10 (100) surfaces of metal oxides (001), denoted MO.

$\delta-\varepsilon$ analysis shows that breaking the $\mathrm{OH}-\mathrm{OOH}$ scaling relation is not an infallible recipe to lower OER overpotentials.

\section{RESULTS AND DISCUSSION}

1. Scaling-Based $\delta$ Optimization. We will focus here on the effect that moderate shifts in the adsorption energies have on the predicted overpotential. We use the scaling-based $\delta$ parameter to tune the adsorption energy of $* \mathrm{OH}\left(\Delta G_{\mathrm{OH}}\right)$, see full details in section S1 in the Supporting Information (SI). Note that the adsorption energies of $* \mathrm{OH}$ and $* \mathrm{OOH}$ scale with those of $* \mathrm{O}$ with a slope of 2 , because the oxygen atom in $* \mathrm{OH}$ lacks 1 electron to fulfill the octet rule while *O lacks two..$^{8,15,17,22,25}$ A positive value of $\delta$ means a weakening of $\Delta G_{\mathrm{OH}}$, and a negative $\delta$ implies its strengthening. Because we follow the aforementioned scaling relations, a change in $\Delta G_{\mathrm{OH}}$ by an amount as large as $\delta$ corresponds to a change in $\Delta G_{O}$ of $2 \delta$ and a change in $\delta$ for $\Delta G_{\mathrm{OOH}}$. The effects of $\delta$ (and $\varepsilon$, which will be defined further below) on the reaction energies in eqs $1-4$ are given in eqs $5-8$ (further information is provided in the SI, section $\mathrm{S} 1$ ). With eqs 5-8 at hand, we can now vary the scaling parameter $\delta$ and find its optimal value for each catalyst such that the corresponding overpotential $\left(\eta_{\mathrm{OER}}\right.$ eq 9$)$ is minimal for the range of $\delta$ values considered.

$$
\begin{aligned}
\Delta G_{1} & =\Delta G_{\mathrm{OH}}+\delta \\
\Delta G_{2} & =\left(\Delta G_{\mathrm{O}}+2 \delta\right)-\left(\Delta G_{\mathrm{OH}}+\delta\right) \\
& =\Delta G_{O}-\Delta G_{\mathrm{OH}}+\delta \\
\Delta G_{3} & =\left(\Delta G_{\mathrm{OOH}}+\delta+\varepsilon\right)-\left(\Delta G_{\mathrm{O}}+2 \delta\right) \\
& =\Delta G_{\mathrm{OOH}}-\Delta G_{\mathrm{O}}-\delta+\varepsilon \\
\Delta G_{4} & =4.92-\Delta G_{\mathrm{OOH}}-\delta-\varepsilon \\
\eta_{\mathrm{OER}} & =\max \left(\Delta G_{1}, \Delta G_{2}, \Delta G_{3}, \Delta G_{4}\right) / \mathrm{e}^{-}-1.23 \mathrm{~V}
\end{aligned}
$$

Here, the reaction energies $\Delta G_{1}, \Delta G_{2}, \Delta G_{3}$, and $\Delta G_{4}$ correspond to those calculated for the catalyst materials shown in Figure 1. ${ }^{16-18,22,33-35}$ The values are presented in 
Table S2a-S6a in the SI. For the optimization procedure, we vary two parameters: $\delta$ and $\varepsilon$. For $\delta$ optimization, $\varepsilon$ is set to zero, so that we first perform $\delta$ optimization, and subsequently $\varepsilon$ optimization. Also, $\delta=0$ and $\varepsilon=0$ render eqs 5-8 identical to the reaction energies in eqs $1-4$. It is also worth noting that the sum of eqs 5-8 is always equal to $4.92 \mathrm{eV}$. A step-by-step explanation of the effects of the $\delta-\varepsilon$ optimization procedure on the reaction energies $\left(\Delta G_{1}\right.$ to $\left.\Delta G_{4}\right)$ and $\eta_{\mathrm{OER}}$ is provided in section $S 1$ of the SI using Co-FGM as an example.

It is important to mention the range of $\delta$ chosen for the optimization procedure. Clearly, large values of $\delta$ may significantly lower the predicted overpotentials. However, it might be unfeasible to weaken or strengthen adsorption energies by values outside the range of $\pm 0.5 \mathrm{eV}$. For instance, it has recently been shown that applying strain to Pt-based catalysts in the range of $\pm 3-5 \%$ changes $\Delta G_{\mathrm{OH}}$ by $\pm 0.2-0.25 \mathrm{eV}^{36,37}$ Thus, we chose the value of $\pm 0.3 \mathrm{eV}$ as a conservative estimate range and note that it has no particular influence in our analysis other than serving as an upper bound to $\delta$ (see further details in Figure S1).

Figure 2a shows the volcano plot before $\delta$ optimization (blue) and after $\delta$ optimization (yellow). We chose $\Delta G_{2}$ as a descriptor in the $x$-axis because it is the most commonly used descriptor in volcano-type activity plots for the OER and has successfully been employed in a number of studies. ${ }^{2,20,22}$ Additionally, a recent study by Viswanathan and co-workers found $\Delta G_{2}$ to be an optimal descriptor for the $4 \mathrm{e}^{-}$OER reaction. ${ }^{24}$ However, several other descriptors can be used, for instance $\Delta G_{\mathrm{O}}{ }^{18} \Delta G_{\mathrm{O}}-$ $\Delta G_{\mathrm{OOH}},{ }^{38}$ the catalysts' bulk energy of formation, ${ }^{34}$ the binding energy of $\mathrm{O}_{2}$ and the redox potentials of the metal centers for molecular catalysts, ${ }^{39}$ and structural parameters such as the $\mathrm{O}$ pband center and the occupation of the $e_{g}$ orbitals for perovskites. $^{40,41}$ The data points in blue are taken from the literature (Figure 1) $16-18,22,33-35$ and the overpotential is calculated using $\Delta G_{\mathrm{O}}, \Delta G_{\mathrm{OH}}, \Delta G_{\mathrm{OOH}}$, and eqs 5-9. Similarly, using the values of $\delta$ in the range of $\pm 0.3 \mathrm{eV}$ (and $\varepsilon=0$ ), the optimized $\eta_{\mathrm{OER}}$ (lowest value of $\eta_{\mathrm{OER}}$ in the range of $\delta$ considered) is computed for all the catalyst materials considered (See section $S 1$ for details) and plotted together with $\Delta G_{2}$ (from eq 6) are plotted again in yellow on the volcano plot. For example, consider porphyrin $\mathrm{Cr}-\mathrm{H}^{18}$ (Table S2a) with $\Delta G_{\mathrm{O}}=$ $0.84 \mathrm{eV}, \Delta G_{\mathrm{OH}}=0.26 \mathrm{eV}, \Delta G_{\mathrm{OOH}}=3.53 \mathrm{eV}$ and $\eta_{\mathrm{OER}}=1.46 \mathrm{~V}$. For this catalyst, $\delta=+0.3 \mathrm{eV}$ renders $\Delta G_{\mathrm{O}}{ }^{\delta}=1.44 \mathrm{eV}, \Delta G_{\mathrm{OH}}{ }^{\delta}=$ $0.56 \mathrm{eV}, \Delta G_{\mathrm{OOH}}{ }^{\delta}=3.83 \mathrm{eV}$ and $\eta_{\mathrm{OER}}{ }^{\delta}=1.16 \mathrm{~V}$ (Table S2b). Therefore, weakening $\Delta G_{\mathrm{OH}}$ by $0.3 \mathrm{eV}$ lowers $\eta_{\mathrm{OER}}$ by $0.3 \mathrm{~V}$.

In Figure 2, applying $\delta$ visibly lowers the overpotentials for most materials, as the points in yellow are shifted upward along both legs of the volcano plot, indicating a decrease in $\eta_{\text {OER }}$ that follows scaling relations. In fact, over $93 \%$ of the catalysts show an improvement in overpotential larger than $0.1 \mathrm{~V}$ upon $\delta$ optimization. The material with the lowest overpotential before $\delta$ optimization is the perovskite $\mathrm{SrCoO}_{3}$, with $\eta_{\mathrm{OER}}=0.31 \mathrm{~V}$, while the catalyst with the lowest overpotential after $\delta$ optimization is Co-FGM with $\eta_{\mathrm{OER}}{ }^{\delta}=0.22 \mathrm{~V}$ (the reason for the change in the material with the lowest overpotential upon $\delta$ optimization is uncovered by the ESSI analysis and discussed in detail in section S2 in the SI), implying that one can go above the top of the volcano $\left(\eta_{\text {OER }}=0.37 \mathrm{~V}\right)$ even with the restrictions imposed by scaling relations, owing to the dispersion in the linear fit of the $\mathrm{OH}-\mathrm{OOH}$ scaling. ${ }^{23,24}$ In other words, if the energetic separation between $* \mathrm{OH}$ and $* \mathrm{OOH}$ is $3.2 \pm 0.2$ $\mathrm{eV},{ }^{15,18,22-24}$ the top of the volcano is located at $0.37 \pm 0.2 \mathrm{~V}$, so (a)

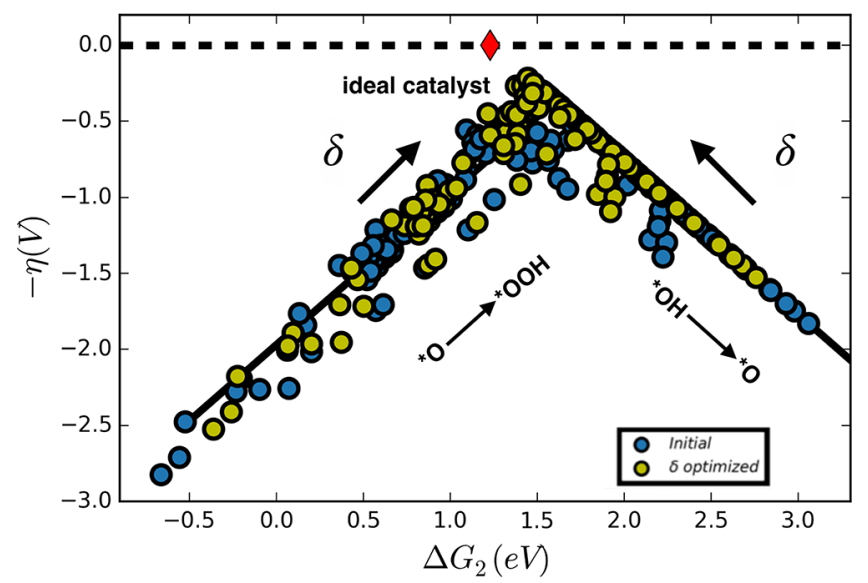

(b)

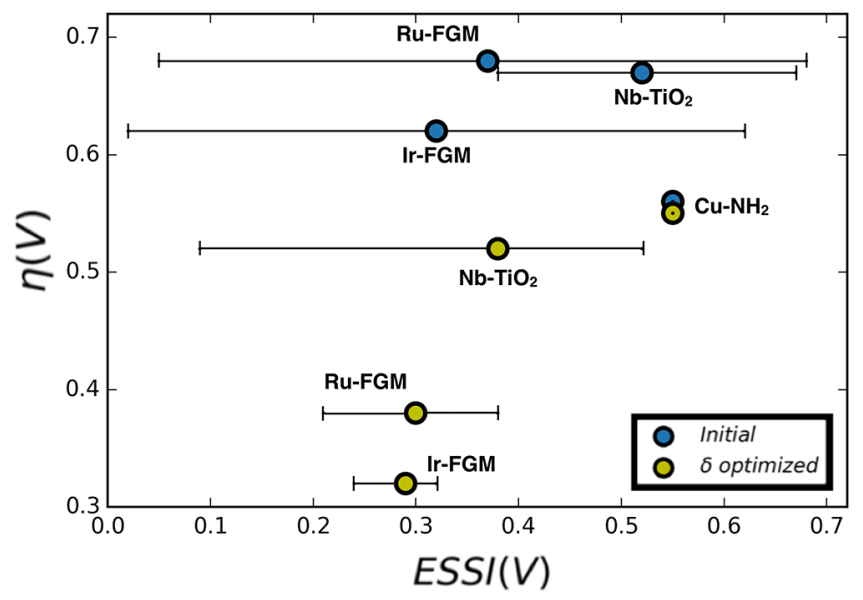

Figure 2. (a) Volcano plot for all the materials (porphyrins, perovskites, monoxides, (un)doped $\mathrm{TiO}_{2}$ and functionalized graphitic materials) considered in this study (see Figure 1) before (blue) and after (yellow) the optimization of the scaling-based parameter $\delta$. (b) Selected examples of materials with different range bars in the ESSI axis, before (blue) and after (yellow) $\delta$ optimization. While materials with high step-symmetry (narrow ESSI range bars) do not benefit from $\delta$ optimization, materials with low step symmetry (large ESSI range bars) show a large reduction in overpotential. The maximal reduction in overpotential is limited by the boundaries of $\delta$, namely $[-0.3,0.3] \mathrm{eV}$.

that with scaling-based optimization one can reach $\eta_{\mathrm{OER}}{ }^{\delta}=0.17$ $\mathrm{V}$.

While the catalysts with high overpotentials benefit the most from $\delta$ optimization, the procedure becomes increasingly less effective at lower overpotentials close to the top of the volcano, as the reaction energies become similar and several of them compete to be potential limiting. To capture this feature of $\delta$ optimization, we resort to the electrochemical step symmetry index (ESSI): ${ }^{42}$

$$
\mathrm{ESSI}=\frac{1}{n} \sum_{i=1}^{n}\left(\frac{\Delta G_{i}^{+}}{\mathrm{e}^{-}}-E^{0}\right)
$$

where $\Delta G_{i}^{+} \geq 1.23 \mathrm{eV}$ come from eqs $5-8$, and $E^{0}=1.23 \mathrm{~V}$ for the OER. In this context, electrocatalytic symmetry is defined with respect to the ideal catalyst, which has $\Delta G_{1}=\Delta G_{2}=\Delta G_{3}=$ $\Delta G_{4}=1.23 \mathrm{eV}$, and, thus, ESSI $=0$.

Consider the metal oxide $\mathrm{NiO}^{33,34}$ (Table S4a), which has $\Delta G_{1}=1.04 \mathrm{eV}, \Delta G_{2}=1.45 \mathrm{eV}, \Delta G_{3}=1.63 \mathrm{eV}$, and $\Delta G_{4}=0.80$ 
$\mathrm{eV}$. Its overpotential is $\eta_{\mathrm{OER}}=(1.63-1.23) \mathrm{eV} / \mathrm{e}^{-}=0.4 \mathrm{~V}$ (from eq 9$)$, and ESSI $=((1.45-1.23)+(1.63-1.23)) / 2=0.31 \mathrm{~V}$ (from eq 10).

Note that if $n>1$ in eq 10, one can add range bars to ESSI to show the dispersion of the data (see Figure $2 b$, Figure $3 b$ and

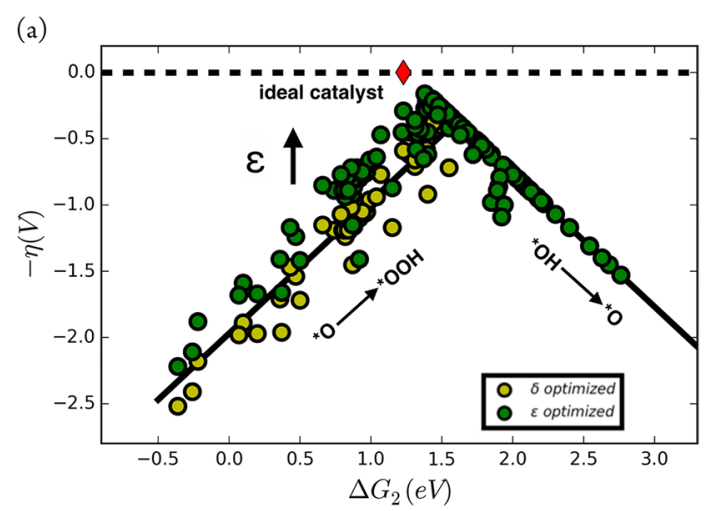

(b)

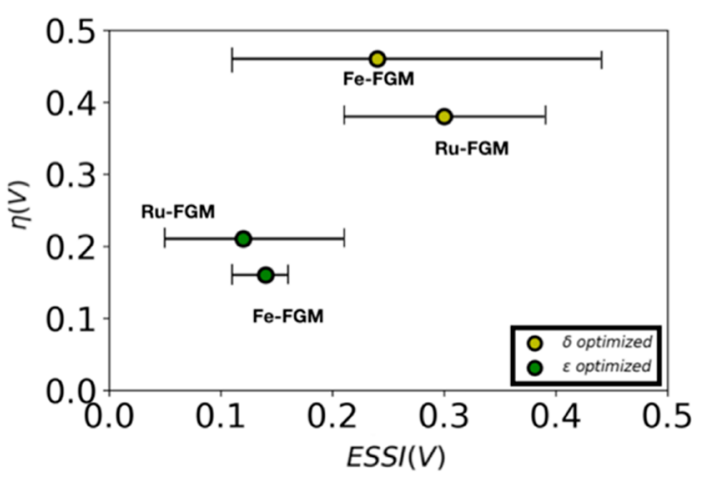

Figure 3. (a) Volcano plot for all the materials (porphyrins, perovskites, monoxides, (un)doped $\mathrm{TiO}_{2}$ and functionalized graphitic materials) considered in this study (see Figure 1 ) after $\delta$ optimization but before (yellow) and after (green) the optimization of the scaling-free parameter $\varepsilon$. (b) Selected examples of materials with different range bars in the ESSI axis after consecutive $\delta$ (yellow) and $\varepsilon$ (green) optimizations. The maximal reduction in overpotential is limited by the boundaries of $\varepsilon$, namely $[-0.3,0] \mathrm{eV}$.

section S2 in the SI). By only considering the reaction energies exceeding the equilibrium potential, ESSI captures the following two facts in Figure 2b:

(1) The ease of optimization of catalysts having the same overpotential can be substantially different, depending on the degree of symmetry of their reaction steps. For instance, the porphyrin $\mathrm{Cu}-\mathrm{NH}_{2}{ }^{18}$ (Table S2a) and IrFGM $^{17,35}$ (Table S5a) have similar $\eta_{\text {OER }}$ overpotentials ( 0.56 and $0.62 \mathrm{~V}$, respectively). While the overpotential reduces by $0.01 \mathrm{~V}$ after $\delta$ optimization for $\mathrm{Cu}-\mathrm{NH}_{2}$, it reduces by $0.3 \mathrm{~V}$ for Ir-FGM (Figure $2 \mathrm{~b}$ ). Thus, the latter is more prone to optimization than the former. While just the adsorption energies and the overpotential do not help to identify the ease of optimization for a given catalyst (as depicted in conventional volcano plots), $\delta$ optimization or ESSI together with its associated range bars allow for such identification. This is illustrated in Figure $2 b$, where the ESSI for $\mathrm{Cu}-\mathrm{NH}_{2}$ is $0.55 \mathrm{~V}$ with a small range bar of 0.01 $\mathrm{V}$, indicating high step symmetry and difficult optimization, whereas the ESSI for Ir-FGM is $0.32 \mathrm{~V}$ with a wide range bar of $0.6 \mathrm{~V}$, indicating asymmetry and ease of optimization. Thus, despite the fact that both catalysts have similar overpotentials, their degree of symmetry provides a measure of their proclivity for optimization.

(2) For a given OER electrocatalyst, the specific electrochemical steps with reaction energies larger than $1.23 \mathrm{eV}$ are important. Based on eqs $5-8$, it can be seen that $\Delta G_{1}$ and $\Delta G_{2}$, and $\Delta G_{3}$ and $\Delta G_{4}$ have the same sign of $\delta$ (positive in the former, negative in the latter), while all other combinations (e.g., $\Delta G_{1}$ and $\Delta G_{4}$, and $\Delta G_{2}$ and $\Delta G_{3}$ ) have opposite $\delta$ signs. The implications of this are that, if a catalyst has combinations of reaction energies that have opposite $\delta$ signs as part of the ESSI, such a catalyst is harder to optimize than a catalyst having reaction free energies with the same $\delta$ signs. Consider $\mathrm{Nb}$ doped $\mathrm{TiO}_{2}{ }^{16}$ (denoted $\mathrm{Nb}-\mathrm{TiO}_{2}$, Table S6a) and graphitic materials with $\mathrm{Ru}-\mathrm{N}_{4}$ active sites ${ }^{17,35}$ (denoted Ru-FGM, Table S5a). Although they have similar $\eta_{\text {OER }}$ ( 0.67 and $0.68 \mathrm{~V}$, respectively), the change in the overpotential after $\delta$ optimization is $0.15 \mathrm{~V}$ for the former and $0.3 \mathrm{~V}$ for the latter (Figure $2 \mathrm{~b}$ ). The reason for this is clear from the reaction energies larger than $1.23 \mathrm{eV}$. For $\mathrm{Nb}-\mathrm{TiO}_{2}$, such energies are $\Delta G_{2}$ and $\Delta G_{3}$ (1.90 and 1.61 $\mathrm{eV})$, whereas they are $\Delta G_{3}$ and $\Delta G_{4}(1.91$ and $1.28 \mathrm{eV})$ for Ru-FGM. Since $\mathrm{Nb}-\mathrm{TiO}_{2}$ has limiting reaction energies with opposite $\delta$ signs, they compete with each other during the optimization procedure to become potential limiting, as one of them increases while the other decreases. This sets a limit on the effect of $\delta$ upon the overpotential. However, for Ru-FGM the reaction energies have identical $\delta$ signs, so that there is no such competition during optimization, resulting in maximum benefit.

The examples considered in Figure $2 b$ illustrate that materials with similar overpotential may have a substantially different ease of optimization, due to the specific reaction energies larger than $1.23 \mathrm{eV}$, namely those contributing to the assessment of ESSI and its associated range bars. All this points toward the fact that one should concentrate on the potential-limiting steps for each catalyst, as done by the ESSI analysis rather than focusing exclusively on the sum of steps 2 and $3\left(\Delta G_{2}+\Delta G_{3}\right)$, which are the steps responsible for the $\mathrm{OH}-\mathrm{OOH}$ constant separation of $3.2 \mathrm{eV}$.

2. Scaling-Free $\boldsymbol{\varepsilon}$ Optimization. Now that we have performed scaling-based $\delta$ optimization, in a second step we introduce the scaling-free parameter $\varepsilon$. This parameter stabilizes the adsorption energy of $* \mathrm{OOH}$ only (see eqs $7-8$ and section S1 in the SI), so by definition $\varepsilon \leq 0$ in all cases. Such scaling-free stabilization has often been suggested in the literature as the route to superior OER electrocatalysts by breaking the $\mathrm{OH}$ $\mathrm{OOH}$ scaling. ${ }^{22,25,26,30,32,43}$ As in the case of $\delta$, to be conservative, we use a range of $[-0.3,0] \mathrm{eV}$ for $\varepsilon$ as it has been shown previously that covalence can help stabilize *OOH in porphyrin systems typically by up to $\sim 0.3 \mathrm{eV} .{ }^{18}$ However, we do not discard the fact that particular cases may go beyond that range. ${ }^{30,31,44-46}$ The main aim of $\varepsilon$ optimization is to examine whether or not all catalysts benefit from breaking the $\mathrm{OH}-\mathrm{OOH}$ scaling relation (see Section S1). Note that we use the $\eta_{\mathrm{OER}}$ obtained after the $\delta$ optimization as a starting point to construct the volcano plot in Figure $3 \mathrm{a}$ and the ESSI activity plots in Figures $3 \mathrm{~b}$ and $4 \mathrm{c}$. 


\section{(a) Initial}

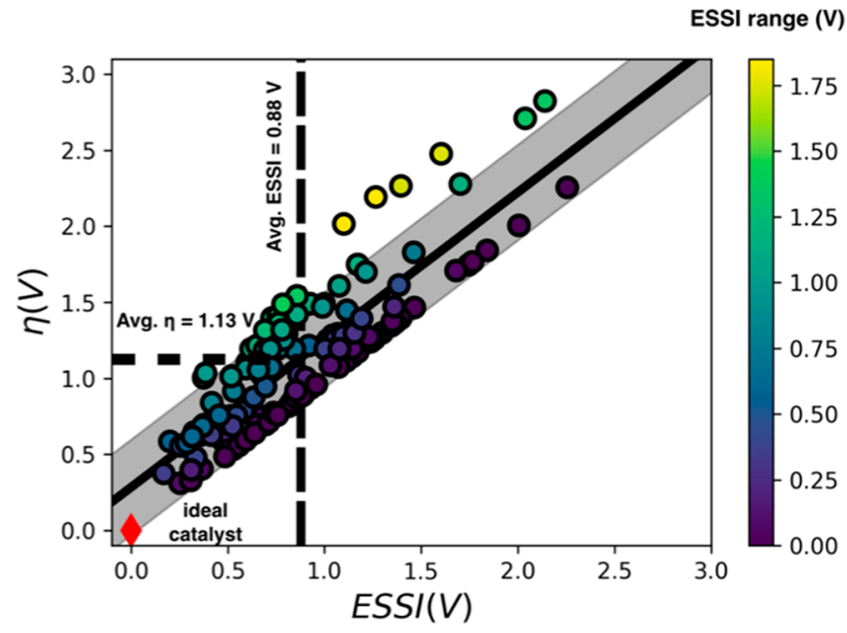

(b) After $\delta$ optimization

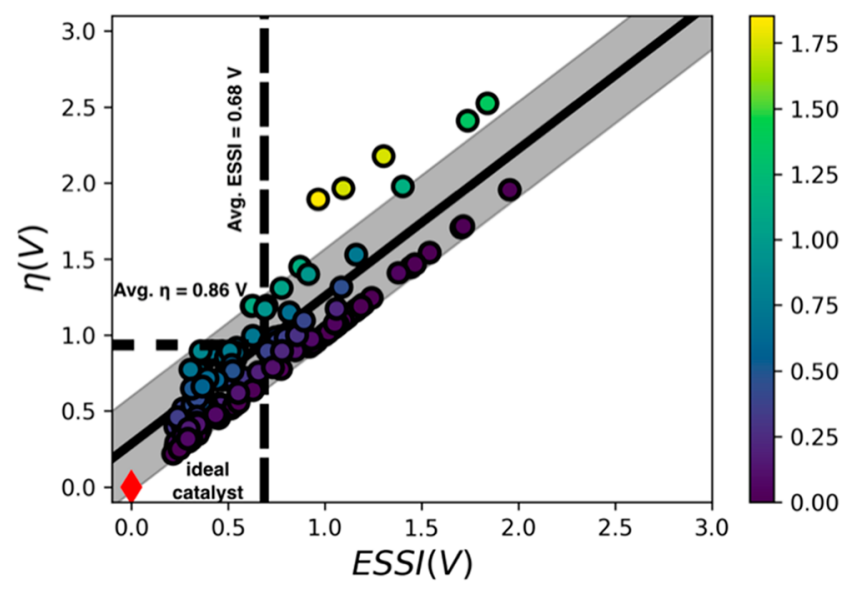

(c) After $\varepsilon$ optimization

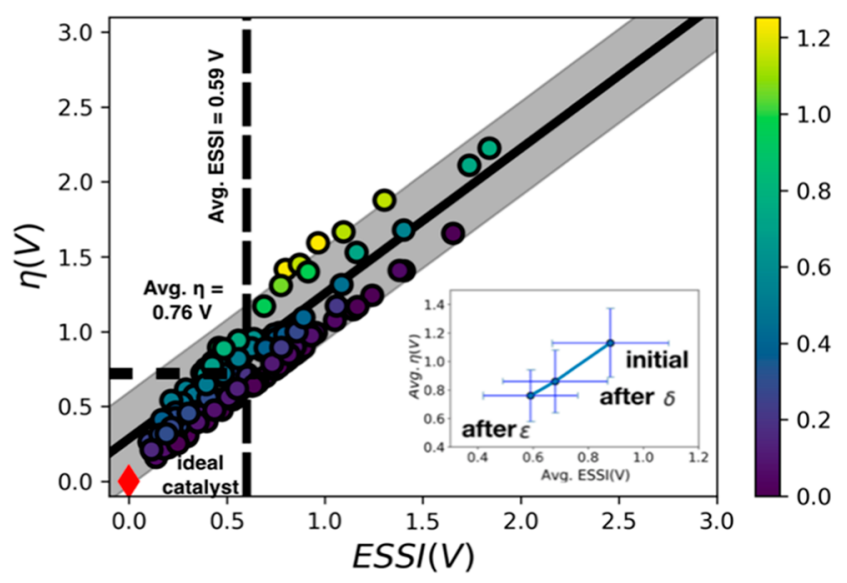

Figure 4. Overpotentials as a function of ESSI. (a) Before any optimization, (b) after $\delta$ optimization, and (c) after $\delta$ and $\varepsilon$ optimizations. The ESSI range is shown on the colored $z$-axis. The dashed lines are the ESSI and $\eta$ values averaged over all materials, which decrease along the optimization procedure, toward the ideal catalyst (inset in c). MAEs of the linear fit also decrease from $0.21 \mathrm{eV}$ (a) to 0.18 $\mathrm{eV}(\mathrm{b})$ and $0.15 \mathrm{eV}(\mathrm{c})$ as a result of the increase in step symmetry throughout the optimization.
For materials that have $\Delta G_{3}(* \mathrm{O} \rightarrow * \mathrm{OOH})$ as the potentiallimiting step, such an optimization should result in the decrease of the overpotential, as $\varepsilon$ reduces the reaction energy of this step (see eq 7). This is observed as a vertical shift of the points on left leg of the volcano plot in Figure 3a, where most of the materials limited by $\Delta G_{3}$ lie. Conversely, most of the points on the right leg remain unaltered, as those correspond mostly to materials limited by $\Delta G_{1}\left(\mathrm{H}_{2} \mathrm{O} \rightarrow{ }^{*} \mathrm{OH}\right)$ or $\Delta G_{2}(* \mathrm{OH} \rightarrow * O)$, where $* \mathrm{OOH}$ is not involved.

Thus, it is clear that (1) $\varepsilon$ optimization (i.e., *OOH stabilization with respect to $* \mathrm{OH}$ ) does not lower the overpotentials of all materials. (2) It only lowers those limited by $\Delta G_{3}$. (3) It deleteriously affects those limited by $\Delta G_{4}$ $\left(* \mathrm{OOH} \rightarrow \mathrm{O}_{2}\right)$, increasing their overpotential. In fact, only $37 \%$ of the analyzed catalysts show an improvement in overpotential $>0.1 \mathrm{~V}$ upon $\varepsilon$ optimization. This is interesting, as $49 \%$ of the data points in Figure 2 are located on the left leg of the volcano.

From $\varepsilon$ optimization we conclude that the reduction in $\eta_{\mathrm{OER}}$ as a result of breaking the $\mathrm{OH}-\mathrm{OOH}$ scaling relation strongly depends on the reaction steps larger than $1.23 \mathrm{eV}$ (i.e., those used to evaluate ESSI). Although a simple, universal rule of thumb is highly desirable in electrocatalysis, for the OER it seems that every material needs separate analyses. In this context, $\varepsilon$ optimization provides a systematic way of assessing the effect of breaking the $\mathrm{OH}-\mathrm{OOH}$ scaling relation on the activity for a given catalyst. Such insight is useful before engaging into the formidable task of trying to break scaling relations.

Another important observation from Figure $3 \mathrm{~b}$ is the reduction in the ESSI range bars after both $\delta$ and $\varepsilon$ optimizations, implying that an increase in the symmetry of the reaction energies is an important attribute of nearly ideal electrocatalysts. For instance, for Fe-FGM, ${ }^{17,35}$ the ESSI range bar spans $0.35 \mathrm{~V}$ after $\delta$ optimization, while it reduces to $0.05 \mathrm{~V}$ after further optimization with $\varepsilon$, as shown in Figure $3 \mathrm{~b}$.

We summarize the most important effects of the $\delta-\varepsilon$ optimization in the ESSI-based activity plots in Figure 4. The average overpotential and ESSI are seen to decrease alongside after each optimization step (inset in Figure 4c), with the decrease being more pronounced for $\delta$ optimization. This implies that a subsequent optimization with the scaling-free parameter $\varepsilon$ enhances fewer materials, as discussed above. In addition, the mean absolute error (MAE) of the linear fit between ESSI and $\eta_{\mathrm{OER}}$ also decreases along the two-step optimization procedure as a result of the increase in step symmetry.

A recent experimental work showed that, by changing the electrolyte for the OER catalyzed by $\mathrm{IrO}_{2}, \Delta G_{\mathrm{OH}}$ can be changed without affecting $\Delta G_{\mathrm{O}} \cdot{ }^{47}$ In other words, one can break the O$\mathrm{OH}$ scaling by suitable electrolyte selection. This is analogous to $\varepsilon$ optimization, as it involves breaking scaling relations, albeit between $* \mathrm{OH} / * \mathrm{O}$ and is termed $\varepsilon^{*}$ optimization (see section S4 for a detailed analysis). A number of materials are seen to benefit more from $\delta-\varepsilon^{*}$ optimization compared to $\delta-\varepsilon$ optimization (Table S1). Therefore, the systematic analyses of $\delta-\varepsilon$ and $\delta-\varepsilon^{*}$ optimization provide valuable insights on the specific scaling relations that have to be broken for a given material and reaction.

3. Benefits of $\delta-\varepsilon$ vs $\varepsilon$ Optimization. It is useful to ask now if there is a particular benefit in performing a two-step $\delta-\varepsilon$ optimization as opposed to just a single-step $\varepsilon$ optimization. In other words, one may wonder at this point whether it is more 
convenient to only break scaling relations or reach first the top of the volcano and then break them.

Figure 5 illustrates the advantage of having a two-step $\delta-\varepsilon$ optimization, using Fe-FGM as an example catalyst. The initial

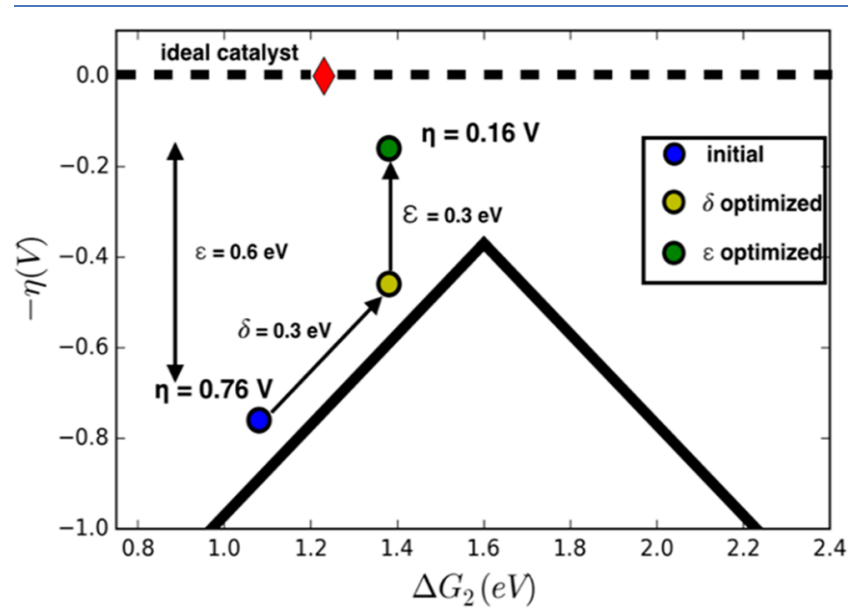

Figure 5. Volcano plot illustrating the difference between moderate $\delta-\varepsilon$ optimization vs extreme $\varepsilon$ optimization using Fe-FGM as an example. While a large $\varepsilon$ value of $0.6 \mathrm{eV}$ is needed to approach the ideal catalyst, a two-step $\delta-\varepsilon$ optimization procedure would only require more realistic values of $0.3 \mathrm{eV}(\delta)$ and $0.3 \mathrm{eV}(\varepsilon)$.

$\eta_{\text {OER }}$ of Fe-FGM is $0.76 \mathrm{~V}$, and it reduces to $0.16 \mathrm{~V}$ after the $\delta-\varepsilon$ optimization. In order to reach this value of $0.16 \mathrm{~V}$ in the volcano plot, $\eta_{\mathrm{OER}}$ has to be reduced by $0.6 \mathrm{~V}$. If a single-step $\varepsilon$ optimization is performed, a large enhancement of $0.6 \mathrm{eV}$ is needed that involves the breaking of the $\mathrm{OH}-\mathrm{OOH}$ scaling, which is an arduous endeavor. ${ }^{25,43}$ Therefore, further optimization with $\delta$ after $\varepsilon$ optimization (i.e., $\varepsilon-\delta$ optimization) although mathematically possible, is challenging from an experimental standpoint, as the two optimization strategies need to be compatible and do not counteract each other. On the other hand, using a two-step $\delta-\varepsilon$ optimization procedure, the reduction of $0.6 \mathrm{~V}$ needed is shared between $\delta(0.3 \mathrm{eV})$ and $\varepsilon$ $(0.3 \mathrm{eV})$, which is more feasible as both values are $<0.5 \mathrm{eV}$. Here it is also worth noting that after $\delta$ optimization the catalyst is already highly active.

All these observations suggest that before trying to break scaling relations, it is recommendable to optimize a catalyst as much as possible in more conventional ways (e.g., ligand, strain effects), $, 36,37,48$ namely using the scaling-based $\delta$ parameter (see section S3). As discussed previously, such an optimization can lead to catalysts with overpotentials as low as $0.22 \mathrm{~V}$ (Co-FGM), noticeably above the top of the volcano at $0.37 \mathrm{~V}$. After the complete optimization of the $\delta$ parameter is achieved, in order to reach the ideal catalyst, scaling relations have to be broken following unconventional routes (nanoconfinement, ligand stabilization, tethering, etc.), ${ }^{25,30,32}$ namely performing $\varepsilon$ optimization.

\section{CONCLUSIONS}

To summarize and conclude, we have presented here guidelines for the optimization of electrocatalysts by introducing into descriptor-based analyses a scaling-based parameter $(\delta)$ and a scaling-free parameter $(\varepsilon)$. The $\delta-\varepsilon$ procedure provides quantitative estimations on the ease of optimization of each catalyst and the particular reaction steps to focus on. Systematic analyses over numerous materials can be made using the ESSI, a descriptor that captures the essence of the $\delta-\varepsilon$ optimization procedure and scales linearly with the predicted overpotentials.

All this was exemplified for the oxygen evolution reaction, where breaking the $\mathrm{OH}-\mathrm{OOH}$ scaling relation is habitually believed to be the key to enhanced electrocatalysis. However, the $\delta-\varepsilon$ optimization mathematically shows that while a large number of materials benefit from scaling-based optimization ( $\sim 93 \%$ of the studied catalysts), only very specific materials can be further optimized by breaking the $\mathrm{OH}-\mathrm{OOH}$ scaling relation (only $\sim 37 \%$ of the studied catalysts).

Instead of using heuristic rules, we hope that future screening studies employ general thermodynamic principles to guide the search for more efficient electrocatalysts.

\section{ASSOCIATED CONTENT}

Supporting Information

The Supporting Information is available free of charge on the ACS Publications website at DOI: 10.1021/acscatal.9b00532.

Optimization procedure using scaling-based $(\delta)$ and scaling-free $(\varepsilon)$ parameters, calculation of ESSI and range bars, $\varepsilon$ optimization analysis, $\delta-\varepsilon^{*}$ optimization analysis, tables with the data used for the $\delta-\varepsilon$ optimization analysis (PDF)

\section{AUTHOR INFORMATION}

\section{Corresponding Author}

*E-mail: f.calle.vallejo@ub.edu.

ORCID $\odot$

Nitish Govindarajan: 0000-0003-3227-5183

Marc T. M. Koper: 0000-0001-6777-4594

Evert Jan Meijer: 0000-0002-1093-9009

Federico Calle-Vallejo: 0000-0001-5147-8635

Notes

The authors declare no competing financial interest.

\section{ACKNOWLEDGMENTS}

N.G. acknowledges funding from NWO and Shell Global International Solutions B.V. under the "Computational Sciences for Energy Research” program, project number 14CSER044 and Project HPC-EUROPA3 (INFRAIA-2016-1-730897), with the support of the EC Research Innovation Action under the H2020 Programme. F.C.V. thanks the Spanish MICIU for a Ramón y Cajal research contract (RYC-2015-18996) and financial support through the program "Units of Excellence María de Maeztu” (grant MDM-2017-0767).

\section{REFERENCES}

(1) Katsounaros, I.; Cherevko, S.; Zeradjanin, A. R.; Mayrhofer, K. J. J. Oxygen Electrochemistry as a Cornerstone for Sustainable Energy Conversion. Angew. Chem., Int. Ed. 2014, 53, 102-121.

(2) Seh, Z. W.; Kibsgaard, J.; Dickens, C. F.; Chorkendorff, I.; Nørskov, J. K.; Jaramillo, T. F. Combining Theory and Experiment in Electrocatalysis: Insights into Materials Design. Science 2017, 355, eaad4998.

(3) Nørskov, J. K.; Rossmeisl, J.; Logadottir, A.; Lindqvist, L.; Kitchin, J. R.; Bligaard, T.; Jónsson, H. Origin of the Overpotential for Oxygen Reduction at a Fuel-Cell Cathode. J. Phys. Chem. B 2004, 108, 1788617892.

(4) Nørskov, J. K.; Bligaard, T.; Rossmeisl, J.; Christensen, C. H. Towards the Computational Design of Solid Catalysts. Nat. Chem. 2009, $1,37$.

(5) Greeley, J.; Stephens, I. E. L.; Bondarenko, A. S.; Johansson, T. P.; Hansen, H. A.; Jaramillo, T. F.; Rossmeisl, J.; Chorkendorff, I.; 
Nørskov, J. K. Alloys of Platinum and Early Transition Metals as Oxygen Reduction Electrocatalysts. Nat. Chem. 2009, 1, 552.

(6) Greeley, J.; Jaramillo, T. F.; Bonde, J.; Chorkendorff, I.; Nørskov, J. K. Computational High-Throughput Screening of Electrocatalytic Materials for Hydrogen Evolution. Nat. Mater. 2006, 5, 909.

(7) Abild-Pedersen, F.; Greeley, J.; Studt, F.; Rossmeisl, J.; Munter, T. R.; Moses, P. G.; Skúlason, E.; Bligaard, T.; Nørskov, J. K. Scaling Properties of Adsorption Energies for Hydrogen-Containing Molecules on Transition-Metal Surfaces. Phys. Rev. Lett. 2007, 99, 16105.

(8) Calle-Vallejo, F.; Martínez, J. I.; García-Lastra, J. M.; Rossmeisl, J.; Koper, M. T. M. Physical and Chemical Nature of the Scaling Relations between Adsorption Energies of Atoms on Metal Surfaces. Phys. Rev. Lett. 2012, 108, 116103.

(9) Calle-Vallejo, F.; Loffreda, D.; Koper, M. T. M.; Sautet, P. Introducing Structural Sensitivity into Adsorption-energy Scaling Relations by Means of Coordination Numbers. Nat. Chem. 2015, 7, 403.

(10) Su, H.-Y.; Sun, K.; Wang, W.-Q.; Zeng, Z.; Calle-Vallejo, F.; Li, W.-X. Establishing and Understanding Adsorption-Energy Scaling Relations with Negative Slopes. J. Phys. Chem. Lett. 2016, 7, 53025306.

(11) Choksi, T.; Majumdar, P.; Greeley, J. P. Electrostatic Origins of Linear Scaling Relationships at Bifunctional Metal/Oxide Interfaces: A Case Study of Au Nanoparticles on Doped MgO Substrates. Angew. Chem., Int. Ed. 2018, 57, 15410-15414.

(12) Koper, M. T. M. Theory of Multiple Proton-electron Transfer Reactions and Its Implications for Electrocatalysis. Chem. Sci. 2013, 4, $2710-2723$

(13) Koper, M. T. M. Thermodynamic Theory of Multi-Electron Transfer Reactions: Implications for Electrocatalysis. J. Electroanal. Chem. 2011, 660, 254-260.

(14) Rossmeisl, J.; Qu, Z.-W.; Zhu, H.; Kroes, G.-J.; Nørskov, J. K. Electrolysis of Water on Oxide Surfaces. J. Electroanal. Chem. 2007, 607, 83-89.

(15) Viswanathan, V.; Hansen, H. A.; Rossmeisl, J.; Nørskov, J. K. Universality in Oxygen Reduction Electrocatalysis on Metal Surfaces. ACS Catal. 2012, 2, 1654-1660.

(16) García-Mota, M.; Vojvodic, A.; Metiu, H.; Man, I. C.; Su, H.-Y.; Rossmeisl, J.; Nørskov, J. K. Tailoring the Activity for Oxygen Evolution Electrocatalysis on Rutile $\mathrm{TiO} 2(110)$ by Transition-Metal Substitution. Chem CatChem 2011, 3, 1607-1611.

(17) Calle-Vallejo, F.; Martínez, J. I.; García-Lastra, J. M.; Abad, E.; Koper, M. T. M. Oxygen Reduction and Evolution at Single-Metal Active Sites: Comparison between Functionalized Graphitic Materials and Protoporphyrins. Surf. Sci. 2013, 607, 47-53.

(18) Calle-Vallejo, F.; Krabbe, A.; García-Lastra, J. M. How Covalence Breaks Adsorption-Energy Scaling Relations and Solvation Restores Them. Chem. Sci. 2017, 8, 124-130.

(19) Vojvodic, A.; Nørskov, J. K. Optimizing Perovskites for the Water-Splitting Reaction. Science 2011, 334, 1355-1356.

(20) Montoya, J. H.; Doyle, A. D.; Nørskov, J. K.; Vojvodic, A. Trends in Adsorption of Electrocatalytic Water Splitting Intermediates on Cubic ABO3 Oxides. Phys. Chem. Chem. Phys. 2018, 20, 3813-3818.

(21) Zhang, B.; Zheng, X.; Voznyy, O.; Comin, R.; Bajdich, M.; García-Melchor, M.; Han, L.; Xu, J.; Liu, M.; Zheng, L.; García de Arquer, F. P.; Dinh, C. T.; Fan, F.; Yuan, M.; Yassitepe, E.; Chen, N.; Regier, T.; Liu, P.; Li, Y.; De Luna, P.; Janmohamed, A.; Xin, H. L.; Yang, H.; Vojvodic, A.; Sargent, E. H. Homogeneously dispersed multimetal oxygen-evolving catalysts. Science 2016, 352, 333-337.

(22) Man, I. C.; Su, H.-Y.; Calle-Vallejo, F.; Hansen, H. A.; Martínez, J. I.; Inoglu, N. G.; Kitchin, J.; Jaramillo, T. F.; Nørskov, J. K.; Rossmeisl, J. Universality in Oxygen Evolution Electrocatalysis on Oxide Surfaces. Chem CatChem 2011, 3, 1159-1165.

(23) Deshpande, S.; Kitchin, J. R.; Viswanathan, V. Quantifying Uncertainty in Activity Volcano Relationships for Oxygen Reduction Reaction. ACS Catal. 2016, 6, 5251-5259.

(24) Krishnamurthy, D.; Sumaria, V.; Viswanathan, V. Maximal Predictability Approach for Identifying the Right Descriptors for Electrocatalytic Reactions. J. Phys. Chem. Lett. 2018, 9, 588-595.
(25) Kulkarni, A.; Siahrostami, S.; Patel, A.; Nørskov, J. K. Understanding Catalytic Activity Trends in the Oxygen Reduction Reaction. Chem. Rev. 2018, 118, 2302-2312.

(26) Song, F.; Bai, L.; Moysiadou, A.; Lee, S.; Hu, C.; Liardet, L.; Hu, $\mathrm{X}$. Transition Metal Oxides as Electrocatalysts for the Oxygen Evolution Reaction in Alkaline Solutions: An Application-Inspired Renaissance. J. Am. Chem. Soc. 2018, 140, 7748-7759.

(27) Li, Y.; Sun, Q. Recent Advances in Breaking Scaling Relations for Effective Electrochemical Conversion of CO2. Adv. Energy Mater. 2016, 6, 1600463.

(28) Peterson, A. A.; Nørskov, J. K. Activity Descriptors for CO2 Electroreduction to Methane on Transition-Metal Catalysts. J. Phys. Chem. Lett. 2012, 3, 251-258.

(29) Back, S.; Jung, Y. Importance of Ligand Effects Breaking the Scaling Relation for Core-Shell Oxygen Reduction Catalysts. ChemCatChem 2017, 9, 3173-3179.

(30) Busch, M.; Halck, N. B.; Kramm, U. I.; Siahrostami, S.; Krtil, P.; Rossmeisl, J. Beyond the Top of the Volcano? - A Unified Approach to Electrocatalytic Oxygen Reduction and Oxygen Evolution. Nano Energy 2016, 29, 126-135.

(31) Halck, N. B.; Petrykin, V.; Krtil, P.; Rossmeisl, J. Beyond the Volcano Limitations in Electrocatalysis - Oxygen Evolution Reaction. Phys. Chem. Chem. Phys. 2014, 16, 13682-13688.

(32) Doyle, A. D.; Montoya, J. H.; Vojvodic, A. Improving Oxygen Electrochemistry through Nanoscopic Confinement. ChemCatChem 2015, 7, 738-742.

(33) Calle-Vallejo, F.; Inoglu, N. G.; Su, H.-Y.; Martínez, J. I.; Man, I. C.; Koper, M. T. M.; Kitchin, J. R.; Rossmeisl, J. Number of Outer Electrons as Descriptor for Adsorption Processes on Transition Metals and Their Oxides. Chem. Sci. 2013, 4, 1245-1249.

(34) Calle-Vallejo, F.; Díaz-Morales, O. A.; Kolb, M. J.; Koper, M. T. M. Why Is Bulk Thermochemistry a Good Descriptor for the Electrocatalytic Activity of Transition Metal Oxides? ACS Catal. 2015, 5, 869-873.

(35) Calle-Vallejo, F.; Martínez, J. I.; Rossmeisl, J. Density Functional Studies of Functionalized Graphitic Materials with Late Transition Metals for Oxygen Reduction Reactions. Phys. Chem. Chem. Phys. 2011, 13, 15639-15643.

(36) Calle-Vallejo, F.; Bandarenka, A. S. Enabling Generalized Coordination Numbers to Describe Strain Effects. ChemSusChem 2018, 11, 1824-1828.

(37) Escudero-Escribano, M.; Malacrida, P.; Hansen, M. H.; VejHansen, U. G.; Velázquez-Palenzuela, A.; Tripkovic, V.; Schiøtz, J.; Rossmeisl, J.; Stephens, I. E. L.; Chorkendorff, I. Tuning the Activity of Pt Alloy Electrocatalysts by Means of the Lanthanide Contraction. Science 2016, 352, 73-76.

(38) Briquet, L. G. V; Sarwar, M.; Mugo, J.; Jones, G.; Calle-Vallejo, F. A New Type of Scaling Relations to Assess the Accuracy of Computational Predictions of Catalytic Activities Applied to the Oxygen Evolution Reaction. ChemCatChem 2017, 9, 1261-1268.

(39) Zagal, J. H.; Koper, M. T. M. Reactivity Descriptors for the Activity of Molecular MN4 Catalysts for the Oxygen Reduction Reaction. Angew. Chem., Int. Ed. 2016, 55 (47), 14510-14521.

(40) Han, B.; Risch, M.; Lee, Y.-L.; Ling, C.; Jia, H.; Shao-Horn, Y. Activity and Stability Trends of Perovskite Oxides for Oxygen Evolution Catalysis at Neutral pH. Phys. Chem. Chem. Phys. 2015, 17, 22576-22580.

(41) Jacobs, R.; Hwang, J.; Shao-Horn, Y.; Morgan, D. Assessing Correlations of Perovskite Catalytic Performance with Electronic Structure Descriptors. Chem. Mater. 2019, 31, 785-797.

(42) Govindarajan, N.; García-Lastra, J. M.; Meijer, E. J.; CalleVallejo, F. Does the Breaking of Adsorption-Energy Scaling Relations Guarantee Enhanced Electrocatalysis? Curr. Opin. Electrochem. 2018, 8, $110-117$.

(43) Vojvodic, A.; Nørskov, J. K. New Design Paradigm for Heterogeneous Catalysts. Natl. Sci. Rev. 2015, 2, 140-143.

(44) Busch, M. Water Oxidation: From Mechanisms to Limitations. Curr. Opin. Electrochem. 2018, 9, 278-284. 
(45) Frydendal, R.; Busch, M.; Halck, N. B.; Paoli, E. A.; Krtil, P.; Chorkendorff, I.; Rossmeisl, J. Enhancing Activity for the Oxygen Evolution Reaction: The Beneficial Interaction of Gold with Manganese and Cobalt Oxides. ChemCatChem 2015, 7, 149-154.

(46) Ping, Y.; Nielsen, R. J.; Goddard, W. A. The Reaction Mechanism with Free Energy Barriers at Constant Potentials for the Oxygen Evolution Reaction at the IrO2 (110) Surface. J. Am. Chem. Soc. 2017, 139, 149-155.

(47) Kuo, D.-Y.; Kawasaki, J. K.; Nelson, J. N.; Kloppenburg, J.; Hautier, G.; Shen, K. M.; Schlom, D. G.; Suntivich, J. Influence of Surface Adsorption on the Oxygen Evolution Reaction on IrO2(110). J. Am. Chem. Soc. 2017, 139, 3473-3479.

(48) Retuerto, M.; Calle-Vallejo, F.; Pascual, L.; Ferrer, P.; García, Á.; Torrero, J.; Gianolio, D.; Fierro, J. L. G.; Peña, M. A.; Alonso, J. A.; Rojas, S. Role of Lattice Oxygen Content and Ni Geometry in the Oxygen Evolution Activity of the Ba-Ni-O System. J. Power Sources 2018, 404, 56-63. 